\title{
Role of ARK5 in cancer and other diseases (Review)
}

\author{
GUOHENG MO $^{1}$, BOHAN ZHANG ${ }^{2}$ and QUNGUANG JIANG ${ }^{3}$ \\ ${ }^{1}$ Department of Neurosurgery, Queen Mary College of Nanchang University; ${ }^{2}$ First Clinical Medical College, \\ The First Affiliated Hospital of Nanchang University; ${ }^{3}$ Department of Gastrointestinal Surgery, \\ The First Affiliated Hospital of Nanchang University, Nanchang, Jiangxi 330006, P.R. China
}

Received September 21, 2019; Accepted June 20, 2020

DOI: $10.3892 /$ etm.2021.10129

\begin{abstract}
Malignant tumors are often exposed to hypoxic and glucose-starved microenvironments. AMP-activated protein kinase (AMPK) is an energy sensor that is stimulated during energy-deficient conditions and protects cells from hypoxic injury by regulating metabolism. AMPK-related protein kinase 5 (ARK5) is a member of the catalytic sub-unit of the AMPK family and has an important role in energy regulation and hypoxia. ARK5 is regulated by Akt and liver kinase B1 and is associated with numerous tumor-related molecules to exert the negative effects of tumors. Studies have revealed ARK5 overexpression in cases of tumor invasion and metastasis and a positive association with the degree of cancer cell malignancy, which is regarded as a key element in determining cancer prognosis. Furthermore, ARK5 downregulation improves drug sensitivity through the epithelial-mesenchymal transition pathway, indicating that it may be a potential therapeutic target. In other non-cancer conditions, ARK5 has various roles in neurodegenerative diseases (Alzheimer's and Huntington's disease), renal disorders (diabetic nephropathy and renal fibrosis) and physiological processes (striated muscle generation). In the present review, the upstream and downstream molecular pathways of ARK5 in cancer and other diseases are described and potential therapeutic strategies are discussed.
\end{abstract}

\section{Contents}

1. Introduction

2. Relevance of ARK5 in cancer

3. Emerging role of ARK5 in tumorigenesis and progression.

4. ARK5 as a potential therapeutic target

Correspondence to: Dr Qunguang Jiang, Department of Gastrointestinal Surgery, The First Affiliated Hospital of Nanchang University, 17 Yongwaizheng, Nanchang, Jiangxi 330006, P.R. China E-mail: fbron.student@sina.com

Key words: AMP-activated protein kinase-related protein kinase 5, tumor metastasis, AMP-activated protein kinase-related protein kinase 5-associated diseases, potential therapeutic strategies, cancer
5. Relationship between ARK5 and other diseases

6. Conclusions and future perspectives

\section{Introduction}

Malignant tumors often feature inadequate angiogenesis, structure and function as well as over-proliferation and increased energy demand when growing in microenvironments with insufficient blood supply (1). When cancer cells proliferate in these harsh conditions, they must adapt by regulating their cell cycles to improve blood flow and adjust the balance of energy metabolism to maintain proliferation. This is a hypoxic reaction and various molecules are involved in this process (2). Cancer cell response in hypoxic conditions has been extensively studied and hypoxia is widely accepted as a specific marker indicating poor cancer prognosis (3).

AMPK-related protein kinase 5 (ARK5) is a serine/threonine kinase that has been identified as the fifth member of the AMP-activated protein kinase (AMPK) family (4). ARK5 serves a role in the metastasis and invasion of colorectal (CRC) cancer, pancreatic cancer (PC), gastric cancer, hepatic cancer and squamous cell carcinoma (5-8). Akt, the most important ARK 5 upstream regulator, phosphorylates ARK5 at the Ser600 residue (a C-terminal site outside the catalytic domain) ans activates $74 \mathrm{kDa}$ kinases (9). Akt is also an important mediator of cancer proliferation, survival and oncogenesis $(9,10)$.

ARK5-mediated Akt was established as a key element that functions as a survival factor in the complex tumorigenesis network (9). ARK5 prevents cell death under hypoxic and glucose-starved conditions by avoiding death receptor (RAS) activation in cells and inhibiting caspase-8 activation by inducing cellular Fas-associated protein with death domain-like interleukin (IL) $1 \beta$-converting enzyme-inhibitory protein (c-FLIP) in cancer cells $(9,11)$. Previous studies have revealed that ARK5 is involved in hypoxia-induced cancer cell tolerance to glucose starvation by regulating the transforming growth factor- $\beta$ (TGF- $\beta$ ) signaling pathway $(12,13)$. Furthermore, ARK5 causes drug resistance by inducing certain cellular morphology transformations, including myosin filament reorganization (13).

As an important and recently investigated intermediate molecule, ARK5 has promising long-term research value. In the current review, the molecular interactions, physical progress and different functions of ARK5 in cancer and 
other diseases, as well as potential therapeutic strategies, are discussed (Fig. 1).

\section{Relevance of ARK5 in cancer}

Multiple myeloma. Multiple myeloma is a common cancer where 20,000 new cases are diagnosed annually in the United States $(14,15)$. The introduction of autologous stem cell transplantation and novel drugs with various mechanisms of action (including proteasome inhibition and immunomodulation) have fundamentally changed the treatment strategy for multiple myeloma and have significantly prolonged the overall survival of patients (16-19). However, despite these advances, patients only survive for 7-8 years after diagnosis due to drug resistance and minimal residual disease (20).

c-Musculoaponeurotic fibrosarcoma (MAF), which is transcription factor that involved in immune responses and works as a $\mathrm{T}$ cell stimulator that induces IL-4 and IL-10 release to control $\mathrm{T}$ cell, was revealed to participate in the regulation of fiber cell differentiation (21-23). c-MAF translocation and overexpression have been reported in numerous cases of multiple myeloma (24-26). In various clinical trials, 351 clinical specimens exhibited clear ARK5 and $\mathrm{c}-\mathrm{MAF}$ overexpression in multiple myeloma-derived cell lines. Sequence analysis of the ARK5 gene promoter further revealed that the gene contained two putative MAF-recognition elements and that ARK5 mRNA acts as a regulator in c-MAF-induced multiple myeloma (8). These results suggested that ARK5 may be a transcriptional target of the large MAF family.

A previous in vitro study has demonstrated that ARK5 expression is a key element in multiple myeloma invasion and metastasis and participates in these processes by modulating insulin-like growth factor (IGF)-1 expression (8). Schiller et al and Perumal et al $(27,28)$ demonstrated that the ratio of AMP/ATP increased during energy deficiency, leading to phosphoric acid-dependent AMPK activation. Eventually, the level of ATP returned to normal due to the inhibition of energy-consuming pathways.

The overexpression of ARK 5 has been previously found to exert negative effects on the apoptosis regulation of multiple myeloma by inducing glucose starvation tolerance (29). ARK5 is directly stimulated by Akt, which regulates cancer cell survival and proliferation $(9-11,30)$. Inhibition of ARK5 can lead to the reduction in ATP levels in cells that abnormally express MYC, leading to a variety of pro-apoptotic reactions (31). MYC is widely known as a strong factor in tumorigenesis $(29,32)$.

In conclusion, AKR5 has become a more promising choice to inhibit malignant tumors and prolong patient survival due to high reversal of drug resistance. ARK5 is closely associated with multiple myeloma and may be an effective therapeutic target.

CRC. CRC is one of the most lethal cancers worldwide (15). An available treatment method for all stages of CRC is tumor resection, with chemotherapy and radiotherapy commonly used as neoadjuvants in locally advanced CRC (33). However, the prognosis of patients with CRC remain poor (33). A total of 241 pairs of cDNA from normal tissues and 13 different tumor specimens from patients with CRC were sequenced via DNA array analysis. The results revealed that ARK5 was overexpressed in CRC. Additionally, a total of 56 clinical specimens of primary CRC and liver metastasis demonstrated high ARK5 expression (31).

Poor clinical prognosis caused by ARK5 is primarily associated with a hypoxic microenvironment (34). Hypoxia is very common in tumors and is associated with proliferation, invasiveness, metastasis and drug resistance (35). Hypoxia-inducible factor (HIF)1 serves a regulatory role and can be used as a key prognostic indicator of tumor hypoxia in CRC (36). HIF-1 is a dimer composed of HIF1- $\alpha$ and HIF1- $\beta$ subunits (37). HIF1- $\alpha$ is a regulator that is triggered by hypoxia and subsequently regulates the activity of the entire complex (37). The expression and absence of HIF1- $\alpha$ is associated with poor prognosis in patients with CRC (38).

Kusakai et al (31) revealed that ARK5 was overexpressed in malignant CRC tumors and that this expression dynamically increased in hypoxic conditions. Additionally, it was determined that ARK5 and HIF1- $\alpha$ were overexpressed in CRC, demonstrating a clear linear correlation. ARK5 is regulated by HIF1- $\alpha$, which amplifies the ARK5 signal and promotes cancer cell survival in hypoxic conditions (37). Due to this, ARK5 is highly expressed in hypoxic solid tumor-associated blood vessels, which is an another important factor in cancer cell angiogenesis and metastasis (37).

HIF1- $\alpha$ expression downstream of ARK5 is associated with tumor stage, tumor grade, lymph node metastasis and liver metastasis (35). However, further research is required to assess the degree of malignancy in solid tumors.

$P C . P C$ is the most fatal cancer and is the eighth leading cause of cancer-related death worldwide (39). Prognosis is often poor due to high invasiveness, rapid proliferation and limited treatment $(15,39)$.

By analyzing a rank-based meta-analysis of individual histological features associated with pancreatic ductal adenocarcinoma (40), 8 genes associated with PC progression were identified: ARK5, E2F transcription factor 3, high mobility group AT-Hook 2, RAS P21 protein activator 1, insulin receptor substrate 1 , actinin $\alpha 1$, Sloan-Kettering oncogene and $\Delta$-like protein 1 pre-cursor. ARK5 is one of the potential regulators that was significantly differentially expressed (40). These results may indicate that AKR5 serves a significant role in PC.

Gemcitabine (GEM) is the sole first-line drug for advanced $\mathrm{PC}$, although it only prolongs patient survival for a few months due to clinical multi-drug resistance (41). Further research into this resistance mechanism is required to improve PC treatment $(15,42,43)$. Studies have demonstrated that hypoxia increases PC cell resistance to GEM-induced apoptosis $(44,45)$. Additionally, under hypoxic conditions, ARK5 inhibition significantly increases the sensitivity of PC cells to GEM (44). In a previous study, the inhibition of ARK5 reversed the effects of hypoxia on E-cadherin and vimentin expression, whichh are markers of epithelial-mesenchymal transition (EMT) (46). Therefore, ARK5 regulates GEM resistance under hypoxic conditions through the EMT process (47).

Furthermore, in vivo and in vitro ARK5 overexpression was demonstrated in PC metastasis and invasion models 


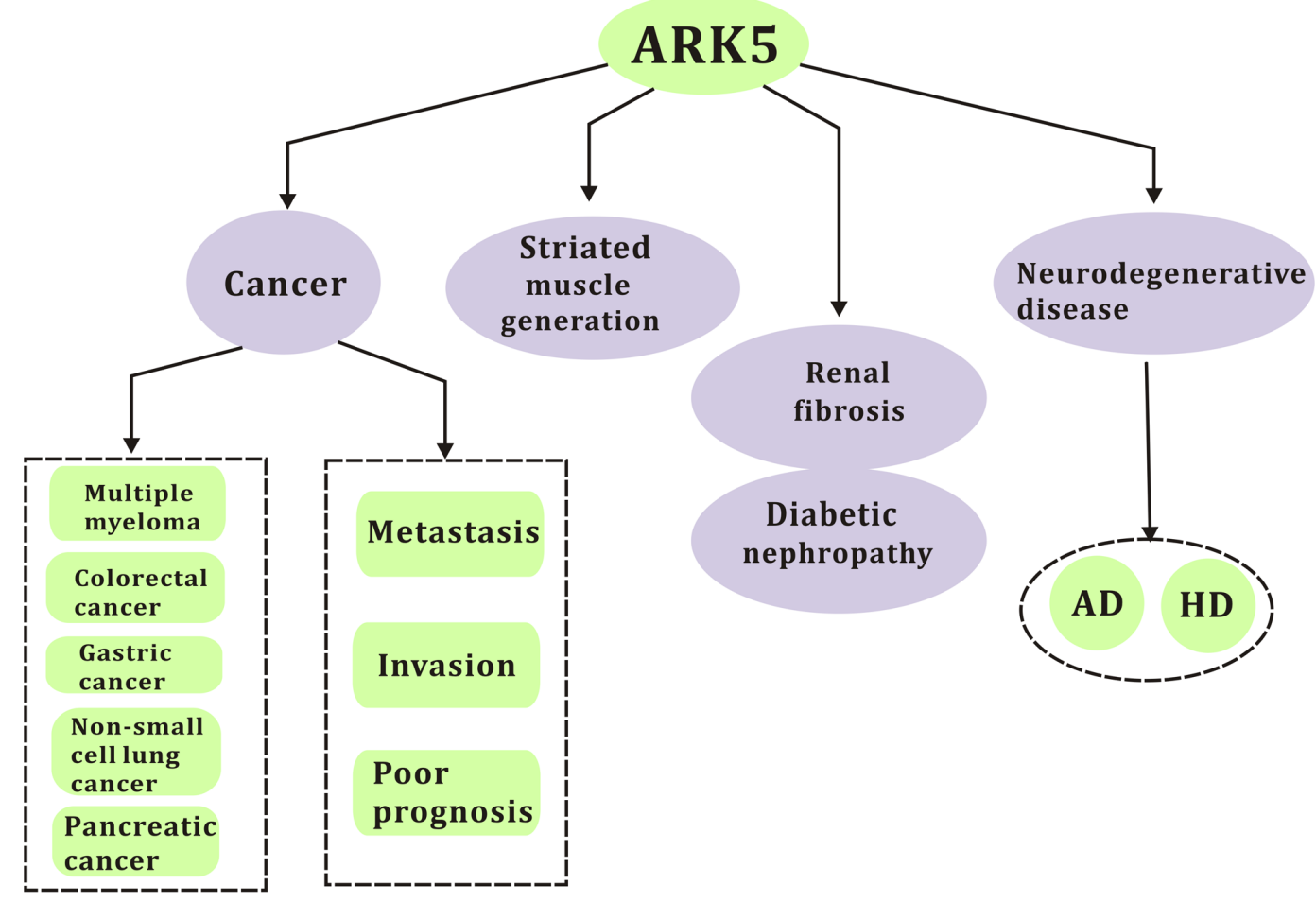

Figure 1. ARK5 serves a role in various diseases. ARK5, AMP-activated protein kinase-related protein kinase 5. AD, Alzheimer's disease; HD, Huntington's disease.

through EMT $(9,10)$. The detection and intervention of ARK5 expression may therefore improve drug sensitivity and improve prognosis and survival time in patients with PC.

Other cancers. ARK5 expression was confirmed in various types of cancer, including osteosarcoma, ovarian, hepatic, CRC, gastric, breast and non-small cell lung cancer (NSCLC). NSCLC is one of the most common malignant tumors worldwide and accounts for $\sim 1 / 3$ of all cancer-related deaths $(48,49)$. Currently, chemotherapy is one of the most effective treatments for NSCLC, with cisplatin being the standard first-line drug, though its long-term therapeutic efficacy reduced by drug resistance (49).

Although various factors are known to induce chemical resistance, the mechanism of cisplatin resistance remains unclear (50). A report has previously indicated that epithelial phenotype NSCLC is more sensitive to chemotherapy compared with a mesenchymal phenotype (51). Mesenchymal tumors that express E-cadherin regain chemical sensitivity (51). In ARK5-knockdown cells, sensitivity to cisplatin increased significantly, suggestomg that it may serve as a potential strategy to improve NSCLC drug resistance. Furthermore, in head and neck squamous cell carcinoma (HNSCC), a study has demonstrated that the expression of micro RNA (miRNA or miR) associated with the invasion and metastasis of melanoma. This was also observed in epithelial ovarian cancer (52).

ARK5 overexpression is associatied with poor prognosis (53-55) and has been identified in various solid tumors. Numerous studies have also demonstrated that ARK5 activation can induce the survival of cancer cells during nutritional deficiency $(8,56)$.

\section{Emerging role of ARK5 in cancer genesis and progression}

ARK5 and Akt. ARK5 is a member of the AMPK family and its highly conserved T loop is phosphorylated by two molecules: Akt kinase, which acts on serine600 and liver kinase (LK) B1, which acts on threonine200 (57). Research has revealed that ARK5 transcription is regulated by $\mathrm{spl}$ transcription-activated protein, metabolic pressure and cofactors required at the sp- 1 site $(58,59)$. This covers almost all the pathways that involve ARK5.

Akt is a serine-threonine protein kinase that functions as a key regulator of cell survival and serves an important role in tumor genesis, cell survival, proliferation and differentiation (60). Accelerated Akt activation in malignant tumors (including invasion and metastasis) is associated with gene amplification in various types of cancer, including CRC, PC, gastric and ovarian cancer (61-63). Therefore, Akt is essential to malignant tumors

Numerous studies have confirmed the role of Akt in promoting. tumor invasion and metastasis $(31,64)$ during nutritional starvation. However, the downstream factors of Akt in these processes have not been determined.

A previous study has indicated that Akt-1 and Akt-2 expression in CRC and liver metastasis is higher compared with normal tissue and that ARK5 is expressed in highly malignant clinical specimens, especially in those with invasive morphology (29). Since it has been demostrated that Akt is the most direct upstream pathway of ARK5 and ARK5 is the only known protein of the AMPK familiy assocuated with the Akt pathway, this would indicate a close regulatory effect between Akt and ARK5 (55).

Furthermore, Akt mediates several cellular responses induced by insulin and IGF, such as glycogen synthesis via 
the phosphorylation of glycogen synthase kinase 3 (65). In a previous study, ARK5 was revealed to mediate the invasion of PC and CRC and to promote cancer cell survival by activating the Akt signaling pathway via IGF-1 (66). Furthermore, nuclear DBF-related kinase 2 (NDR2) was revealed to be activated by IGF-1 treatment, phosphorylating threonine 211 on the active T loop of ARK5 (56). ARK5 was also demonstrated to be downstream of NDR2 during activation of IGF-1 signaling (67). Therefore, ARK5 promotes cancer cell survival under the regulation of Akt and ARK5 serves an important role in hypoxia and the Akt pathway in apoptosis in various types of cancer.

The RAF-MEK-ERK and PI3K-Akt-HIF- $\alpha$ pathways serve an important role in cancer development as they are downstream of RAS (68), a protein that regulates cancer cell survival. Akt, as the most direct downstream regulator of RAS, regulates the downstream factor HIF1- $\alpha$ (69). Previous studies have determined a close association between the HIF1- $\alpha$-mediated RAS pathway and ARK5 (68-70).

A linear correlation was reported between ARK5 and HIF1- $\alpha$ expression $(68,70)$. In a previous study, short interfering RNA suppressed HIF1- $\alpha$ expression under hypoxic conditions. The results revealed that the protein and mRNA levels of ARK5 were significantly decreased indicating that ARK is regulated by HIF1- $\alpha$ and that ARK5 lie downstream of HIF1- $\alpha$ under hypoxic conditions where HIF1- $\alpha$ amplifies the role of ARK5 in hypoxia (57). In conclusion, ARK5 is the key gene of HIF1- $\alpha$-mediated cancer proliferation and migration under hypoxic conditions.

ARK5 overexpression was demonstrated to significantly stimulate the invasiveness and metastasis of $\mathrm{PC}$ cells in vitro and in vivo by activating matrix metallopeptidase (MMP) and matrix metalloproteinase 1 (MT1)-MMP (7). MMPs, especially MMP-2 and MMP-9, participate in tumor metastasis and MT1-MMP is the most common activating agent of these (34,71). Research has demonstrated that ARK5 increased MMP-2 and MMP-9 production levels and induced their activation via MT1-MMP production (7).

Davis et al (58) previously hypothesized that ARK5 activation served as a metabolic checkpoint in the regulation of apoptosis, cell cycle progression and arrest, which confirmed ARK5-modulated 'glucose metabolism' as the most significantly aberrantly affected cellular signaling pathway in a model system for highly metastatic tumors.

ARK5 and EMT. EMT is involved in numerous biological and pathological processes, is associated with chemotherapeutic resistance and has an invasive and anti-apoptotic role in cancer tissues (72). EMT is the process by which polar epithelial cells with firm cell-cell adhesion transform into mesenchymal cells with highly invasive capacity (73). At the molecular level, the gene expression of these cells undergoes numerous changes: The expression of epithelial genes (E-cadherin, tight junction protein 1 and occludin) decreases and the expression of mesenchymal genes (N-cadherin, vimentin and fibronectin) increases (74).

In a study on epithelial ovarian cancer, ARK5 was highly expressed in cancer cells compared with normal tissue and was revealed to be strongly associated with EMT (51). Furthermore, ARK5 was reported to regulate the progression of EMT in various solid tumors (74-78). When ARK5 expression was decreased in cells, the resultant inhibition of E-cadherin expression and the downregulation of vimentin expression were related to ARK5 activation (79).

Since the recurrence of E-cadherin expression was demonstrated to increase the sensitivity of cancer tissues to chemotherapeutic agents in various studies, reducing the expression of ARK5 may increase sensitivity to drugs such as doxorubicin (dox) and cisplatin (75,78).

TGF- $\beta 1$ is a key induction factor of the EMT pathway in tumors (80) and induces EMT in NSCLC (81). ARK5 knockdown was reported to decrease TGF- $\beta 1$-induced EMT and invasion and metastasis of cells under hypoxic conditions (82). The results indicated that ARK5 may be involved in the hypoxia-induced TGF- $\beta 1$ pathway in cancer cells, which contributes to glucose starvation tolerance (82). In a liver study, ARK5 expressed lipid fibers and ultimately induced hepatic cell necrosis $(52,83)$. This process is also observed in PC (84). ARK5 inhibits cancer cell death stimulation and promotes normal tissue necrosis. Additionally, another study reported that the suppression of ARK5 reversed hypoxia-induced EMT in hepatic cancer cells (81). The results suggested that ARK5 overexpression is indicative of hypoxia. An additional protein, zinc finger E-box-binding homeobox 1 (ZEB1), also acts as an activator of EMT in mantle cell lymphoma cells and determines resistance to different chemotherapeutic drugs (81). ARK5 was demonstrated to suppress ZEB1 to improve drug sensitivity $(81,85)$.

In conclusion, ARK5 is associated with drug resistance in solid tumors and mediates the EMT pathway and numerous EMT-related molecules such as TGF- $\beta 1$ and ZEB1. Since ARK5 knockdown improves resistance to clinical drugs, ARK5 may serve as a new target to reverse drug resistance.

ARK5 and Fas. Fas is a member of the tumor necrosis factor receptor family (86). As a transmembrane protein, it transmits apoptotic signals in cells and induces apoptosis when Fas ligands bind to the Fas receptor (87). Fas is widely expressed in normal tissues and tumors.

Fas-cell mediated apoptosis serves an important role in various biological processes as it triggers a series of downstream pathways $(86,87)$, suggesting that ARK5 inhibits the activation of caspase- 8 and the expression of caspase- 6 containing two putative ARK5 phosphorylation sites: Ser80 and Ser257 (29), and ultimately inhibits the phosphorylation induced by Fas ligand and Fas. This could promote the survival of cancer cells under conditions of nutritional starvation $(12,86)$.

In further studies, ARK5 was revealed to inhibit caspase-8 activation by preserving c-FLIP in response to Akt stimulation $(4,88)$, preventing cell death caused by glucose starvation and RAS activation in cancer cells $(8,86)$. RAS-induced apoptosis was demonstrated to be inhibited by increased ARK5 expression (11). These results suggested that ARK5 is associated with energy metabolism and cell apoptosis.

ARK5 and MYC. MYC is an important inducer protein in cancer that interferes with cell cycle metabolism and ribosome synthesis (89). When combined with MYC-associated factor X, MYC regulates transcription and oncogenic activity (90). MYC 
overexpression has been demonstrated to induce cell apoptosis as it cannot maintain an adequate ratio of ATP/ADP $(91,92)$. Kusakai et $\mathrm{al}$ and Cox and Der $(67,68)$ previously demonstrated that, using synthetic lethal RNAi screening, ARK5 kinases regulated protein expression by activating various pathways that ultimately maintained or triggered cancer cell survival, particularly when MYC was overexpressed.

Activation of ARK5 and AMPK in response to metabolic stress combats apoptosis in cancer cells and they are markers for most solid tumors $(93,94)$. AMPK is activated by the tumor inhibitor LKB1 (95). In the absence of LKB1, AMPK responds to calcium ions by phosphorylating calcium/calmodulin-dependent protein kinase kinase $2(95,96)$. As a member of the AMPK family, ARK5 is also primarily activated by LKB1 (55).

However, Ciccarese et al (97) revealed a second pathway that maintains ARK5 activity in the absence of LKB1: ARK5 responds to calcium signaling through protein kinase $\mathrm{C} \alpha(\mathrm{PKC} \alpha)$ to regulate AMPK activation (98) and mTORC1-dependent protein translation, protecting cells from MYC-driven apoptosis.

The Calcium-AMPK-mTORC1 metabolic checkpoint-dependent activation requires PKC $\alpha$ and ARK5 while in the absence of ARK5, activated mTOR increases ATP consumption and impairs MYC response to AMPK (98). Therefore, in the presence of ARK5, ATP synthesis is enhanced through the MYC pathway. The depletion of PKC $\alpha$ and ARK5 leads to apoptosis, which suggests that this pathway serves an active role in tumor maintenance (29). The results indicated a novel role for calcium ions in supporting cancer cell viability and elucidated the synthetic lethal interaction between ARK5 and MYC (98). Similarly, PKC $\alpha$ and $-\beta$ have been demonstrated to phosphorylate Akt $(99,100)$, preventing typical MYC-induced apoptosis by inhibiting the expression and function of apoptotic Bcl-2 homology protein (100-102). Therefore, ARK 5 expression is necessary for MYC overexpression, even if Akt is overexpressed (29), suggesting that ARK5 may be a potential target for treating MYC-driven cancer $(4,103)$.

Overall, ARK5 and PKC $\alpha$ may control multiple pathways that promote cancer cell survival. The targeted suppression of these pathways may therefore have potential therapeutic benefits in numerous types of cancer in which MYC is deregulated (103).

At the organelle level, ARK5 is involved in energy regulation by maintaining mitochondrial adaptability and stability and increases the expression of proteins in the respiratory chain by activating MYC, which enhances respiratory capacity (55). When ARK5 is depleted, this phenomenon is eliminated (55). As an important upstream regulator of MYC, ARK5 serves an important role in tumor regulation at the organelle level.

ARK5 and aneuploidy. The AMPK family has 13 different sub-groups with different regulatory modes (104). However, all of them are activated by LKB1 under conditions of metabolic pressure, when ATP levels are low (105). Tumor protein P53 is a downstream protein of AMPK and its phosphorylation serves an important role in apoptosis and cell aging (105).

ARK 5 is part of the AMPK subfamily. It was demonstrated experimentally that ARK5 regulates P53 phosphorylation in vivo and in vitro, directly interacting with the P53 nucleus under the regulation of LKB1 (106). Additionally, ARK5 activation via $\mathrm{P} 21$ and weak acid resistance protein 1 prevents cells from entering the $\mathrm{S}$ phase from the G1 phase (106).

ARK5 was demonstrated to induce premature cell aging, which is closely associated with genetic aneuploidy, which had been previously demonstated in fibroblast (89). ARK5 regulates ploidy and senescence. Decreased ARK5 prevents aneuploidy in cells and enhance their replicative lifespan, while increased ARK5 induces gross aneuploidies and senescence (89).

This ARK5-induced aneuploidy increased the genomic instability of cancer cells and allowed them to overgrow, invade and metastasize, demonstrating the role of ARK5 in tumor regulation (89). Similarly to how cancer develops and exacerbates, genomic instability tends to induce cell senescence (107-109). Genomic instability often manifests as an increase in aneuploidy and a decrease in large tumor suppressor kinase 1 (LATS1) expression, a kinase involved in mitotic exit (109). Decreased LATS1 levels block cell division, affect genomic stability and increase the amount of abnormal DNA in each cell (109). AMPK accelerates P53-mediated cell senescence and ARK5 also leads to genomic aneuploidy changes without the involvement of P53 (110). However, experimental knockdown or overexpression of ARK5 did not affect cellular P53 activity (110).

In summary, in terms of cellular senescence regulation, ARK5 knockdown extends the lifespan of cells, metabolism and slows fibroblast senescence in normal individuals, even in the absence of LKB1. LATS1 is a regulator of stable gene expression and the overexpression of ARK5 weakens the expression of LATS1 (89). Such changes are independent of P53, which highlights the potential role of aneuploidy in ARK5-mediated senescence (89), and suggests a difference between the ARK5 and AMPK families at downstream action sites.

Furthermore, ARK5-induced aneuploidy leads directly to the death of MCF10a immortal cells rather than to senescence in other types of cells (89), indicating that aneuploidy serves a different role in different cells.

ARK5 and miRNA. miRNAs are non-coding RNAs 18-22 nucleotides in length that regulate various physiological activities through specific binding to the 3'-untranslated regions (3'UTR) of mRNA (111). miRNAs are involved in cell proliferation, invasion and metastasis (111). Currently, different miRNA families serve different roles in malignant tumors (112). For example, the miR-200 family (miR-200a, -200b, -200c, -141 and -429) slowed EMT in cancer cells by lowering ZEB1/ZEB2 expression $(111,113)$.

It has been demonstrated that ARK5 was regulated by different miRNAs in different cancers and that ARK5 was negatively associated with the expression of certain miRNAs (112). In a liver cancer study, miR-204 reduced ARK5 expression and invasion, and reversed drug resistance (114). Similarly, miR-145 acted as a negative regulator of intrahepatic cholangiocarcinoma via the regulation of ARK5. miR-145 also reduced MT1-MMP, MMP-2 and MMP-9 expression to alter cell metastasis (115). miR-211 is associated with cell invasion and response to melanoma adhesion (116). In HNSCC, miR-203 regulated EMT by targeting ARK5 and 
miR-96 regulated PC malignancy in the same manner (117). These three RNAs function upstream of ARK5 to block cell invasion (112). Overall, ARK5-associated miRNA could be regarded as a potential therapeutic target in the future treatment of cancer.

\section{ARK5 as a potential therapeutic target}

While chemotherapy is still a major strategy in cancer treatment, drug resistance has become a novel limitation that has led to its failure in long-term use (118). Therefore, there is an urgent requirement to elucidate novel strategies to increase drug efficacy.

Salinomycin. It has been established that ARK5 is highly expressed after treatment with certain clinical therapeutic drugs, including dox, 5-fluorouracil and cisplatin (50). Since ARK5 is associated with cancer cell drug resistance, its downregulation may serve as a potential therapeutic strategy to increase drug sensitivity (50). To increase drug sensitivity, salinomycin may be used as it targets ARK5 (50).

Salinomycin is an ionophore antibiotic that kills cancer stem cells and reverses EMT (50). A previous study on lung cancer demonstrated that salinomycin increased dox sensitivity and demonstrated a synergistic effect (combination index, 0.430 with dox) (50). Furthermore, it was revealed that salinomycin suppresses ZEB1, an important EMT pathway molecule $(83,84)$. Therefore, salinomycin may be a novel drug that could be used in drug combinations. However, weight loss and nerve injury are side effects of salinomycin treatment and may affect its application in a clinical setting (119).

Additionally, it has been demonstrated that combination treatment reversed dox-induced EMT morphology, reversed EMT marker protein (vimentin and E-cadherin) expression and inhibited ARK5 expression (75). This process was also demonstrated in breast cancer, gastric cancer, non-small cell lung cancer, cholangiocarcinoma and hepatic cancer (120).

ON123300. ON123300 is a novel second-generation oral drug and CDK inhibitor, which exerts dual inhibitory effects on CDK4 and ARK5 (121). Compared with first-generation drugs, the site-specific ON123300 reverses the poor curative effects exerted by other drugs and the resultant poor cancer prognosis, which was demonstrated in multiple myeloma treatment (120). ON123300 was also revealed to exert beneficial effects in breast cancer, glioma and mantle cell lymphomas in vivo and in vitro without obvious harm to normal tissue (122).

ARK5 is a molecule that may lead to first-generation drug failure $(20,28,122)$. However, while ARK5 overexpression has been observed in primary multiple myeloma cells, ON123300 caused cell cycle arrest and apoptosis, serving as an ARK5 and CDK4 inhibitor $(32,28)$. MYC-CDK4 binding in multiple myeloma cells is a key interaction in tumorgenesis and ON123300 also has an impact on the MYC pathway as well as on the retinoblastoma/mTOR pathway (122). Furthermore, ARK5 knockdown significantly increased first-generation drug sensitivity of multiple myeloma $(123,124)$.

$7 x$. As $7 \mathrm{x}$ is a novel cyanopyridopyrimidine compound that acts as a multi-kinase inhibitor, $7 \mathrm{x}$ targets CDK4/cyclin D1 and ARK5 kinases (122). ARK5 is also negatively regulated by $7 x$ (124). A previous study demonstrated that ARK5 targeting resulted in high efficacy when cancer cell proliferation and metastasis were inhibited with no significant signs of toxicity (122).

With the increasing number of compounds that have been discovered as effective drugs targeting the ARK5 protein for cancer treatment, the current study hypothesizes that ARK5 could be considered a significant therapeutic strategy for cancer under $7 x$ treatment.

HTH-01-015. HTH-01-015 is a highly selective protein kinase inhibitor, which mainly affects ARK5 by inhibiting the phosphorylation of myosin phosphatase target subunit 1 (MYPT1), a substrate of ARK5 (125). Experimentally, HTH-01-015 was revealed to slow mitosis by inhibiting the phosphorylation of MYPT1 and preventing the entry of cells into the M phase by regulating DNA replication in the $S$ phase (126). This indicated that HTH-01-015 inhibited ARK5 and modulated cell migration and adhesion. Additionally, HTH-01-015 was revealed to detect the physiological function of ARK5 abnormalities, further elucidating its role (126).

In summary, HTH-01-015 was demonstrated to regulate the physiological functions of cells by acting on ARK5 and could potentially serve as a novel drug to combat clinical drug resistance.

\section{Relationship between ARK5 and other diseases}

ARK5, diabetic nephropathy and renal fibrosis. ARK5 is associated with Tau protein stability. This may explain the potential link between ARK5 and a range of diseases, including neurodegenerative diseases and diabetes (55).

ARK5 serves an important role in human diabetic nephropathy (DN) along with TGF- $\beta 1(127,128)$. DN is often accompanied by a series of renal diseases in which TGF- $\beta 1$ mediates glomerular sclerosis and tubular fibrosis (127).

In vitro, TGF- $\beta 1$ was used to induce a model of renal tubular fibrosis. The protein changes that occur during the EMT process in epithelial cells include: E-cadherin (epithelial) to $\mathrm{N}$-cadherin (mesenchymal) transformation and increased vimentin, $\alpha$-smooth muscle actin, connective tissue growth factor and Notch ligand Jagged-1 (129). By silencing ARK5 and thereby lowering TGF- $\beta 1$ expression, fibrosis was reversed in renal tubular cells. This also influenced downstream proteins of TGF- $\beta 1$ by preventing the stimulation of Jagged-1, verifying the link between ARK5 and EMT $(130,131)$.

ARK5 and striated muscle generation. ARK5 protein was not previously identified in murine skeletal muscle. However, in later studies, ARK5 was demonstrated to be highly expressed in cardiac muscle and skeletal muscle, and ARK5 mRNA was identified via reverse transcription-quantitative PCR (130). Muscle contraction increased ARK5 phosphorylation (132). However, this did not alter ARK5 activity, indicating that phosphorylation of ARK5 at Ser400 cannot stimulate this kinase (132).

Uncordinated-82 (UNC-82) kinase serves a key role in Caenorhabditis elegans, a nematode, and is an orthologue of human ARK5 and SNF1/AMP kinase-regulated kinase (SNARK) that is necessary for myosin filament reorganization 
during cellular elongation (27). Research has suggested that ARK5 may exert a similar function to UNC-82 in striated muscle development (130). ARK5 knockout could lead to alterations in contractile apparatus protein (the actin-myosin cytoskeleton) phosphorylation and SNARK reduction may lead to muscle mass disruption with increasing age. These results revealed the conserved role of UNC-82/ARK5/SNARK in muscle generation across diverse animal lineages (27).

Additionally, ARK5 was demonstrated to be a direct target of large musculoaponeurotic fibrosarcoma proteins and its activation was mediated by MAF-recognition element sequences (133). Activation of ARK5 in muscle allows it to interact with several types of myosin phosphatases (134). Furthermore, the ARK5-MYPT1-serine/threonine-protein phosphatase 1B complex promoted the interaction between ARK5 and 1433 protein, which inhibited phosphatase activity (134).

Ultimately, ARK5 controls cell adhesion and as a regulator of myosin phosphatase compounds; it prevents the phosphorylation of AMPK targets by LKB1 and controls phosphatase compounds to influence the phosphorylation of its targets (135).

Furthermore, experiments have demonstrated that ARK5 is involved in the negative feedback regulation of insulin signaling transduction and inhibits insulin-mediated glucose uptake in skeletal muscle (136).

ARK5 and neurodegenerative diseases. The effects of ARK5 in neurological diseases primarily manifest in three aspects: Promoting the polarization and migration of neurons, affecting the expression and accumulation of Tau protein and affecting neuron apoptosis through CASP6 (137).

Neuronal axons need energy to grow and branch, and this energy comes from the large consumption of ATP in the mitochondria (138). As a mediating factor, ARK5 regulates the growth of axons and cortical neuron branches. The LKB1-ARK5 pathway controls the fixation of mitochondria in axons and ARK5 mediates axon growth and growth of cortical neuron branches (138). Overexpression of ARK5 can increase the axon branch, and knockout can cause growth stagnation (138).

Numerous neurodegenerative proteinopathies share a common pathogenesis: Abnormal accumulation of diseaserelated proteins (139). ARK5 was revealed to regulate Tau by stabilizing the protein via specific Ser356 phosphorylation and its inhibition inhibited Tau expression in Drosophila neurodegeneration (139). The results suggested that reducing Tau expression is potentially an effective strategy to alleviate Tau-related neurodegenerative changes. Furthermore, ARK5 may serve as a new entry point for the treatment of Tau-associated diseases (139).

CASP6 was demonstrated to be an important molecule in neurodegenerative diseases, particularly in Alzheimer's disease (AD) and Huntington's disease (137). Research has revealed that avoiding destruction of CASP6 protected mice from neural diseases, such as AD (140). This protection is the result of ARK5 phosphorylating CASP6 at Ser257, which caused CASP6 inhibition and prevented neural cell death (86). The phosphorylation achieved by AKR5 is indirect and is mediated via the downregulation of p53 expression (106) due to the direct relationship between p53 and CASP6 (141). Furthermore, ARK5-mediated CASP6 phosphorylation inhibited its activation, mediating
CASP6 activity (86). This phosphorylation site is specific for CASP6 (86,142).

The aforementioned study offered a potential site for future drug discovery by targeting this unique site to gradually inhibit neural death. Since ARK5 is a negative regulator of CASP6, a potential strategy could involve activating ARK5 expression or interfering with CASP6 phosphorylation at this specific site.

\section{Conclusion and future perspectives}

The current review summarized all known functions of ARK5 and potential drugs from a limited study size. Being a member of the AMPK family, ARK5 serves a metabolic role through its expression: Promoting the survival of cancer cells in harsh microenvironments. Additionally, ARK5 serves an important role in cell adhesion and metastasis (84).

Various processes are involved in cancer cell drug resistance and further research into this resistance mechanism is required to fully elucidate it. As an emerging molecule, ARK5 exerts multiple functions in certain diseases, particularly in malignant tumors. Since AKR5 can be used to evaluate the malignancy of tumors, metastasis and drug restistancy, it is a potential therapeutic target for cancer and drug resistance. Targeting ARK5 in combination with other drugs could potentially improve drug resistance and inhibit tumor metastasis.

The current review hypothesized that in the future, drugs targeting ARK5 will have impactful and specific effects that will improve clinical drug resistance. However, further research into the role of ARK5 in normal cells may be beneficial as it could reduce the targeting side effects of ARK5 therapy. The present review also hypothesized that the relationship between ARK5 and TAU stability is conducive to further elucidating the molecular mechanism of neurodegenerative diseases in future studies.

\section{Acknowledgements}

Not applicable.

\section{Funding}

This work was supported by the National Natural Science Foundation of China (no. 81560397). But it was neither enrolled in the design of the study, collection, analysis, and interpretation of the data, writing of the report, nor in the decision to submit the article for publication. Every author had full access to all data of the study, and the corresponding author had final responsibility for the decision to submit the article for publication.

\section{Availability of data and materials}

The datasets used and analyzed during the current study are available from the corresponding author on reasonable request.

\section{Author's contributions}

GM and QJ contributed to the concept and design of this manuscript. BZ contributed to data reviewing and part of the 
analysis GM, QJ and BZ wrote the manuscript. All authors read and approved the final version of the manuscript.

\section{Ethics approval and consent to participate}

Not applicable.

\section{Patient consent for publication}

Not applicable.

\section{Competing interests}

The authors declare that they have no competing interests.

\section{References}

1. Esumi H, Izuishi K, Kato K, Hashimoto K, Kurashima Y, Kishimoto A, Ogura $\mathrm{T}$ and Ozawa T: Hypoxia and nitric oxide treatment confer tolerance to glucose starvation in a 5'-AMP-activated protein kinase-dependent manner. J Biol Chem 277: 32791-32798, 2002.

2. Ivanov S, Liao SY, Ivanova A, Danilkovitch-Miagkova A, Tarasova N, Weirich G, Merrill MJ, Proescholdt MA, Oldfield EH, Lee J, et al: Expression of hypoxia-inducible cell-surface transmembrane carbonic anhydrases in human cancer. Am J Pathol 158: 905-919, 2001.

3. Kaluz S, Kaluzova M, Chrastina A, Olive PL, Pastoreková S, Pastorek J, Lerman MI and Stanbridge EJ: Lowered oxygen tension induces expression of the hypoxia marker MN/carbonic anhydrase IX in the absence of hypoxia-inducible factor 1 alpha stabilization: A role for phosphatidylinositol 3'-kinase. Cancer Res 62: 4469-4477, 2002.

4. Suzuki A, Kusakai G, Kishimoto A, Lu J, Ogura T, Lavin MF and Esumi H: Identification of a novel protein kinase mediating Akt survival signaling to the ATM protein. J Biol Chem 278: 48-53, 2003.

5. Li B, Tsao SW, Li YY, Wang X, Ling MT, Wong YC, He QY and Cheung AL: Id-1 promotes tumorigenicity and metastasis of human esophageal cancer cells through activation of PI3K/AKT signaling pathway. Int J Cancer 125: 2576-2585, 2009.

6. Ohta T, Isobe M, Takahashi T, Saitoh-Sekiguchi M, Motoyama T and Kurachi $\mathrm{H}$ : The Akt and ERK activation by platinum-based chemotherapy in ovarian cancer is associated with favorable patient outcome. Anticancer Res 29: 4639-4647, 2009.

7. Renton A, Llanos S and Lu X: Hypoxia induces p53 through a pathway distinct from most DNA-damaging and stress-inducing agents. Carcinogenesis 24: 1177-1182, 2003.

8. Suzuki A, Iida S, Kato-Uranishi M, Tajima E, Zhan F, Hanamura I, Huang Y, Ogura T, Takahashi S and Ueda R: ARK5 is transcriptionally regulated by the Large-MAF family and mediates IGF-1-induced cell invasion in multiple myeloma: ARK5 as a new molecular determinant of malignant multiple myeloma. Oncogene 24: 6936-6944, 2005.

9. Simon PO Jr, McDunn JE, Kashiwagi H, Chang K, Goedegebuure PS, Hotchkiss RS and Hawkins WG: Targeting AKT with the proapoptotic peptide, TAT-CTMP: A novel strategy for the treatment of human pancreatic adenocarcinoma. Int J Cancer 125: 942-951, 2009.

10. Kato K, Ogura T, Kishimoto A, Minegishi Y, Nakajima N, Miyazaki M and Esumi H: Critical roles of AMP-activated protein kinase in constitutive tolerance of cancer cells to nutrient deprivation and tumor formation. Oncogene 21: 6082-6090, 2002.

11. Izuishi K, Kato K, Ogura T, Kinoshita T and Esumi H: Remarkable tolerance of tumor cells to nutrient deprivation: Possible new biochemical target for cancer therapy. Cancer Res 60: 6201-6207, 2000.

12. Suzuki A, Kusakai G, Kishimoto A, Lu J, Ogura T and Esumi H: ARK5 suppresses the cell death induced by nutrient starvation and death receptors via inhibition of caspase 8 activation, but not by chemotherapeutic agents or UV irradiation. Oncogene 22: 6177-6182, 2003
13. Suzuki A, Kusakai G, Shimojo Y, Chen J, Ogura T, Kobayashi M and Esumi $\mathrm{H}$ : Involvement of transforming growth factor-beta 1 signaling in hypoxia-induced tolerance to glucose starvation. J Biol Chem 280: 31557-31563, 2005.

14. Kuehl WM and Bergsagel PL: Molecular pathogenesis of multiple myeloma and its premalignant precursor. J Clin Invest 122: 3456-3463, 2012.

15. Siegel R, Naishadham D and Jemal A: Cancer statistics, 2013. CA Cancer J Clin 63: 11-30, 2013.

16. Benboubker L, Dimopoulos MA, Dispenzieri A, Catalano J, Belch AR, Cavo M, Pinto A, Weisel K, Ludwig H, Bahlis N, et al: Lenalidomide and dexamethasone in transplant-ineligible patients with myeloma. N Engl J Med 371: 906-917, 2014.

17. Rajkumar SV, Blood E, Vesole D, Fonseca R and Greipp PR; Eastern Cooperative Oncology Group: Phase III clinical trial of thalidomide plus dexamethasone compared with dexamethasone alone in newly diagnosed multiple myeloma: A clinical trial coordinated by the Eastern Cooperative Oncology Group. J Clin Oncol 24: 431-436, 2006.

18. Richardson PG, Weller E, Lonial S, Jakubowiak AJ, Jagannath S, Raje NS, Avigan DE, Xie W, Ghobrial IM, Schlossman RL, et al: Lenalidomide, bortezomib, and dexamethasone combination therapy in patients with newly diagnosed multiple myeloma. Blood 116: 679-686, 2010.

19. San Miguel JF, Schlag R, Khuageva NK, Dimopoulos MA, Shpilberg O, Kropff M, Spicka I, Petrucci MT, Palumbo A, Samoilova OS, et al: Bortezomib plus melphalan and prednisone for initial treatment of multiple myeloma. N Engl J Med 359: 906-917, 2008.

20. Kumar SK, Dispenzieri A, Lacy MQ, Gertz MA, Buadi FK, Pandey S, Kapoor P, Dingli D, Hayman SR, Leung N, et al: Continued improvement in survival in multiple myeloma: Changes in early mortality and outcomes in older patients. Leukemia 28: 1122-1128, 2014.

21. Kawauchi S, Takahashi S, Nakajima O, Ogino H, Morita M, Nishizawa M, Nishizawa M, Yasuda $\mathrm{K}$ and Yamamoto M: Regulation of lens fiber cell differentiation by transcription factor c-Maf. J Biol Chem 274: 19254-19260, 1999.

22. Kim JI, Li T, Ho IC, Grusby MJ and Glimcher LH: Requirement for the c-Maf transcription factor in crystallin gene regulation and lens development. Proc Natl Acad Sci USA 96: 3781-3785, 1999.

23. Ring BZ, Cordes SP, Overbeek PA and Barsh GS: Regulation of mouse lens fiber cell development and differentiation by the Maf gene. Development 127: 307-317, 2000.

24. Zhang Z, Tong J, Tang X, Juan J, Cao B, Hurren R, Chen G, Taylor P, Xu X, Shi CX, et al: The ubiquitin ligase HERC4 mediates c-Maf ubiquitination and delays the growth of multiple myeloma xenografts in nude mice. Blood 127: 1676-1686, 2016.

25. Wang S, Juan J, Zhang Z, Du Y, Xu Y, Tong J, Cao B, Moran MF, Zeng Y and Mao X: Inhibition of the deubiquitinase USP5 leads to c-Maf protein degradation and myeloma cell apoptosis. Cell Death Dis 8: e3058, 2017.

26. Qiang YW, Ye S, Chen Y, Buros AF, Edmonson R, van Rhee F, Barlogie B, Epstein J, Morgan GJ and Davies FE: MAF protein mediates innate resistance to proteasome inhibition therapy in multiple myeloma. Blood 128: 2919-2930, 2016.

27. Schiller NR, Duchesneau CD, Lane LS, Reedy AR, Manzon ER and Hoppe PE: The Role of the UNC-82 protein kinase in organizing myosin filaments in striated muscle of caenorhabditis elegans. Genetics 205: 1195-1213, 2017.

28. Perumal D, Kuo PY, Leshchenko VV, Jiang Z, Divakar SK, Cho HJ, Chari A, Brody J, Reddy MV, Zhang W, et al: Dual targeting of CDK4 and ARK5 using a novel kinase inhibitor ON123300 exerts potent anticancer activity against multiple myeloma. Cancer Res 76: 1225-1236, 2016.

29. Liu L, Ulbrich J, Müller J, Wüstefeld T, Aeberhard L, Kress TR, Muthalagu N, Rycak L, Rudalska R, Moll R, et al: Deregulated MYC expression induces dependence upon AMPK-related kinase 5. Nature 483: 608-612, 2012.

30. Hoppe S, Bierhoff H, Cado I, Weber A, Tiebe M, Grummt I and Voit R: AMP-activated protein kinase adapts rRNA synthesis to cellular energy supply. Proc Natl Acad Sci USA 106: 17781-17786, 2009.

31. Kusakai G, Suzuki A, Ogura T, Miyamoto S, Ochiai A, Kaminishi M and Esumi H: ARK5 expression in colorectal cancer and its implications for tumor progression. Am J Pathol 164: 987-995, 2004.

32. Li X, Zhang XA, Li X, Xie W and Huang S: MYC-mediated synthetic lethality for treating tumors. Curr Cancer Drug Targets 15: 99-115, 2015. 
33. Colon Cancer Treatment $\left(\mathrm{PDQ}^{\circledR}\right)$ : Patient Version. PDQ Cancer Information Summaries. Bethesda, MD, 2002.

34. Peng JK, Shen SQ, Wang J, Jiang HW and Wang YQ: Hypoxia-inducible factor 1- $\alpha$ promotes colon cell proliferation and migration by upregulating AMPK-related protein kinase 5 under hypoxic conditions. Oncol Lett 15: 3639-3645, 2018.

35. Semenza GL: Defining the role of hypoxia-inducible factor 1 in cancer biology and therapeutics. Oncogene 29: 625-634, 2010.

36. Semenza GL: Targeting HIF-1 for cancer therapy. Nat Rev Cancer 3: 721-732, 2003.

37. Wang GL, Jiang BH, Rue EA and Semenza GL: Hypoxia-inducible factor 1 is a basic-helix-loop-helix-PAS heterodimer regulated by cellular O2 tension. Proc Natl Acad Sci USA 92: 5510-5514, 1995.

38. Wang JS, Jing CQ, Shan KS, Chen YZ, Guo XB, Cao ZX Mu LJ, Peng LP, Zhou ML and Li LP: Semaphorin 4D and hypoxia-inducible factor-1alpha overexpression is related to prognosis in colorectal carcinoma. World J Gastroenterol 21: 2191-2198, 2015

39. Sirri E, Castro FA, Kieschke J, Jansen L, Emrich K, Gondos A, Holleczek B, Katalinic A, Urbschat I, Vohmann C and Brenner H: Recent trends in survival of patients with pancreatic cancer in Germany and the United States. Pancreas 45: 908-914, 2016.

40. Rajamani D and Bhasin MK: Identification of key regulators of pancreatic cancer progression through multidimensional systems-level analysis. Genome Med 8: 38, 2016.

41. Von Hoff DD, Ervin T, Arena FP, Chiorean EG, Infante J, Moore M, Seay T, Tjulandin SA, Ma WW, Saleh MN, et al: Increased survival in pancreatic cancer with nab-paclitaxel plus gemcitabine. N Engl J Med 369: 1691-1703, 2013.

42. Berlin JD, Catalano P, Thomas JP, Kugler JW, Haller DG and Benson AB III: Phase III study of gemcitabine in combination with fluorouracil versus gemcitabine alone in patients with advanced pancreatic carcinoma: Eastern Cooperative Oncology Group Trial E2297. J Clin Oncol 20: 3270-3275, 2002.

43. Oettle H, Richards D, Ramanathan RK, van Laethem JL, Peeters M, Fuchs M, Zimmermann A, John W, Von Hoff D, Arning M and Kindler HL: A phase III trial of pemetrexed plus gemcitabine versus gemcitabine in patients with unresectable or metastatic pancreatic cancer. Ann Oncol 16: 1639-1645, 2005.

44. Wang Y, Kuramitsu Y, Tokuda K, Baron B, Kitagawa T, Akada J, Maehara S, Maehara Y and Nakamura K: Gemcitabine induces poly (ADP-ribose) polymerase-1 (PARP-1) degradation through autophagy in pancreatic cancer. PLoS One 9: e109076. 2014.

45. Gilkes DM, Semenza GL and Wirtz D: Hypoxia and the extracellular matrix: Drivers of tumour metastasis. Nat Rev Cancer 14 430-439, 2014

46. Wu J, Yang B, Zhang Y, Feng X, He B, Xie H, Zhou L, Wu J and Zheng S: miR-424-5p represses the metastasis and invasion of intrahepatic cholangiocarcinoma by targeting ARK5. Int J Biol Sci 15: 1591-1599, 2019.

47. Wang $X$, Song Z, Chen F, Yang $X$, Wu B, Xie S, Zheng X Cai Y, Chen W and Zhong Z: AMPK-related kinase 5 (ARK5) enhances gemcitabine resistance in pancreatic carcinoma by inducing epithelial-mesenchymal transition. Am J Transl Res 10: 4095-4106, 2018.

48. Jemal A, Murray T, Samuels A, Ghafoor A, Ward E and Thun MJ Cancer statistics, 2003. CA Cancer J Clin 53: 5-26, 2003.

49. Schiller JH, Harrington D, Belani CP, Langer C, Sandler A, Krook J, Zhu J and Johnson DH; Eastern Cooperative Oncology Group: Comparison of four chemotherapy regimens for advanced non-small-cell lung cancer. N Engl J Med 346: 92-98, 2002.

50. Li Y, Qi K, Zu L, Wang M, Wang Y and Zhou Q: Anti-apoptotic brain and reproductive organ-expressed proteins enhance cisplatin resistance in lung cancer cells via the protein kinase B signaling pathway. Thorac Cancer 7: 190-198, 2016.

51. Zhang HY, Li JH, Li G and Wang SR: Activation of ARK5/miR-1181/HOXA10 axis promotes epithelial-mesenchymal transition in ovarian cancer. Oncol Rep 34: 1193-1202, 2015.

52. Yu HG, Wei W, Xia LH, Han WL, Zhao P, Wu SJ, Li WD and Chen W: FBW7 upregulation enhances cisplatin cytotoxicity in non-small cell lung cancer cells. Asian Pac J Cancer Prev 14: 6321-6326, 2013

53. Chang XZ, Yu J, Liu HY, Dong RH and Cao XC: ARK5 is associated with the invasive and metastatic potential of human breast cancer cells. J Cancer Res Clin Oncol 138: 247-254, 2012.

54. Cui J, Yu Y, Lu GF, Liu C, Liu X, Xu YX and Zheng PY: Overexpression of ARK5 is associated with poor prognosis in hepatocellular carcinoma. Tumour Biol 34: 1913-1918, 2013.
55. Sun X, Gao L, Chien HY, Li WC and Zhao J: The regulation and function of the NUAK family. J Mol Endocrinol 51: R15-R22, 2013.

56. Suzuki A, Ogura T and Esumi H: NDR2 acts as the upstream kinase of ARK5 during insulin-like growth factor-1 signaling. J Biol Chem 281: 13915-13921, 2006.

57. Lizcano JM, Göransson O, Toth R, Deak M, Morrice NA, Boudeau J, Hawley SA, Udd L, Mäkelä TP, Hardie DG and Alessi DR: LKB1 is a master kinase that activates 13 kinases of the AMPK subfamily, including MARK/PAR-1. EMBO J 23: 833-843, 2004

58. Davis LE, Jeng S, Svalina MN, Huang E, Pittsenbarger J, Cantor EL, Berlow N, Seguin B, Mansoor A, McWeeney SK and Keller C: Integration of genomic, transcriptomic and functional profiles of aggressive osteosarcomas across multiple species. Oncotarget 8: 76241-76256, 2017.

59. Ryu S and Tjian R: Purification of transcription cofactor complex CRSP. Proc Natl Acad Sci USA 96: 7137-7142, 1999.

60. Datta SR, Brunet A and Greenberg ME: Cellular survival: A play in three Akts. Genes Dev 13: 2905-2927, 1999.

61. Itoh N, Semba S, Ito M, Takeda H, Kawata S and Yamakawa M: Phosphorylation of Akt/PKB is required for suppression of cancer cell apoptosis and tumor progression in human colorectal carcinoma. Cancer 94: 3127-3134, 2002.

62. Nicholson KM and Anderson NG: The protein kinase B/Akt signalling pathway in human malignancy. Cell Signal 14: 381-395, 2002.

63. Ruggeri BA, Huang L, Wood M, Cheng JQ and Testa JR: Amplification and overexpression of the AKT2 oncogene in a subset of human pancreatic ductal adenocarcinomas. Mol Carcinog 21: 81-86, 1998 .

64. Higuchi M, Masuyama N, Fukui Y, Suzuki A and Gotoh Y: Akt mediates $\mathrm{Rac} / \mathrm{Cdc} 42$-regulated cell motility in growth factor-stimulated cells and in invasive PTEN knockout cells. Curr Biol 11: 1958-1962, 2001

65. Lawlor MA and Alessi DR: PKB/Akt: A key mediator of cell proliferation, survival and insulin responses? J Cell Sci 114: 2903-2910, 2001.

66. Taatjes DJ, Naar AM, Andel F III, Nogales E and Tjian R Structure, function, and activator-induced conformations of the CRSP coactivator. Science 295: 1058-1062, 2002

67. Kusakai G, Suzuki A, Ogura T, Kaminishi M and Esumi H: Strong association of ARK5 with tumor invasion and metastasis. J Exp Clin Cancer Res 23: 263-268, 2004.

68. Cox AD and Der CJ: The dark side of Ras: Regulation of apoptosis. Oncogene 22: 8999-9006, 2003.

69. Suzuki A, Lu J, Kusakai G, Kishimoto A, Ogura T and Esumi H: ARK5 is a tumor invasion-associated factor downstream of Akt signaling. Mol Cell Biol 24: 3526-3535, 2004

70. Xie M, Wu X, Zhang J, Zhang J and Li X: Ski regulates Smads and TAZ signaling to suppress lung cancer progression. Mol Carcinog 56: 2178-2189, 2017.

71. Sato H, Takino T, Okada Y, Cao J, Shinagawa A, Yamamoto E and Seiki M: A matrix metalloproteinase expressed on the surface of invasive tumour cells. Nature 370:61-65, 1994.

72. Kaufhold S and Bonavida B: Central role of Snaill in the regulation of EMT and resistance in cancer: A target for therapeutic intervention. J Exp Clin Cancer Res 33: 62, 2014.

73. Fischer KR, Durrans A, Lee S, Sheng J, Li F, Wong ST, Choi H, El Rayes T, Ryu S, Troeger J, et al: Epithelial-to-mesenchymal transition is not required for lung metastasis but contributes to chemoresistance. Nature 527: 472-476, 2015.

74. Thiery JP and Sleeman JP: Complex networks orchestrate epithelial-mesenchymal transitions. Nat Rev Mol Cell Biol 7: 131-142, 2006.

75. Li M, Zheng C, Xu H, He W, Ruan Y, Ma J, Zheng J, Ye C and Li W: Inhibition of AMPK-related kinase 5 (ARK5) enhances cisplatin cytotoxicity in non-small cell lung cancer cells through regulation of epithelial-mesenchymal transition. Am J Trans Res 9: 1708-1719, 2017.

76. Liu Y, Du F, Zhao Q, Jin J, Ma X and Li H: Acquisition of 5-fluorouracil resistance induces epithelial-mesenchymal transitions through the Hedgehog signaling pathway in HCT-8 colon cancer cells. Oncol Lett 9: 2675-2679, 2015.

77. Mallini P, Lennard T, Kirby J and Meeson A: Epithelial-tomesenchymal transition: What is the impact on breast cancer stem cells and drug resistance. Cancer Treat Rev 40: 341-348, 2014.

78. Xu T, Zhang J, Chen W, Pan S, Zhi X, Wen L, Zhou Y, Chen BW, Qiu J, Zhang Y, et al: ARK5 promotes doxorubicin resistance in hepatocellular carcinoma via epithelial-mesenchymal transition. Cancer Lett 377: 140-148, 2016. 
79. Chen D, Liu G, Xu N, You X, Zhou H, Zhao X and Liu Q: Knockdown of ARK5 expression suppresses invasion and metastasis of gastric cancer. Cell Physiol Biochem 42: 1025-1036, 2017.

80. Liotta LA: Tumor invasion and metastases-role of the extracellular matrix: Rhoads memorial award lecture. Cancer Res 46: 1-7, 1986.

81. Chen XF, Zhang HJ, Wang HB, Zhu J, Zhou WY, Zhang H, Zhao MC, Su JM, Gao W, Zhang L, et al: Transforming growth factor- $\beta 1$ induces epithelial-to-mesenchymal transition in human lung cancer cells via PI3K/Akt and MEK/Erk1/2 signaling pathways. Molr Biol Reps 39: 3549-3556, 2012.

82. Park NR, Cha JH, Jang JW, Bae SH, Jang B, Kim JH, Hur W, Choi JY and Yoon SK: Synergistic effects of CD44 and TGF- $\beta 1$ through AKT/GSK-3 $\beta / \beta$-catenin signaling during epithelial-mesenchymal transition in liver cancer cells. Biochem Biophys Res Commun 477: 568-574, 2016.

83. Shiraki K, Tsuji N, Shioda T, Isselbacher KJ and Takahashi H: Expression of Fas ligand in liver metastases of human colonic adenocarcinomas. Proc Natl Acad Sci USA 94: 6420-6425, 1997

84. Yoong KF, Afford SC, Randhawa S, Hubscher SG and Adams DH: Fas/Fas ligand interaction in human colorectal hepatic metastases: A mechanism of hepatocyte destruction to facilitate local tumor invasion. Am J Pathol 154: 693-703, 1999.

85. Sánchez-Tilló E, Fanlo L, Siles L, Montes-Moreno S, Moros A, Chiva-Blanch G, Estruch R, Martinez A, Colomer D, Győrffy B, et al: The EMT activator ZEB1 promotes tumor growth and determines differential response to chemotherapy in mantle cell lymphoma. Cell Death Differ 21: 247-257, 2014.

86. Suzuki A, Kusakai G, Kishimoto A, Shimojo Y, Miyamoto S Ogura T, Ochiai A and Esumi H: Regulation of caspase- 6 and FLIP by the AMPK family member ARK5. Oncogene 23: 7067-7075, 2004

87. Restifo NP: Not so Fas: Re-evaluating the mechanisms of immune privilege and tumor escape. Nat Med 6: 493-495, 2000.

88. Suzuki A, Kusakai G, Kishimoto A, Minegichi Y, Ogura T and Esumi $\mathrm{H}$ : Induction of cell-cell detachment during glucose starvation through F-actin conversion by SNARK, the fourth member of the AMP-activated protein kinase catalytic subunit family. Biochem Biophys Res Commun 311: 156-161, 2003.

89. Conacci-Sorrell M, McFerrin L and Eisenman RN: An overview of MYC and its interactome. Cold Spring Harb Perspect Med 4 a014357, 2014

90. Stine ZE, Walton ZE, Altman BJ, Hsieh AL and Dang CV: MYC, metabolism, and cancer. Cancer Discov 5: 1024-1039, 2015.

91. Evan GI, Christophorou M, Lawlor EA, Ringshausen I, Prescott J, Dansen T, Dansen T, Finch A, Martins C and Murphy D: Oncogene-dependent tumor suppression: Using the dark side of the force for cancer therapy. Cold Spring Harb Symp Quant Biol 70: 263-273, 2005.

92. Murphy DJ, Junttila MR, Pouyet L, Karnezis A, Shchors K, Bui DA, Brown-Swigart L, Johnson L and Evan GI: Distinct thresholds govern Myc's biological output in vivo. Cancer Cell 14: 447-457, 2008

93. Hanahan D and Weinberg RA: Hallmarks of cancer: The next generation. Cell 144: 646-674, 2011.

94. Ross FA, MacKintosh C and Hardie DG: AMP-activated protein kinase: A cellular energy sensor that comes in 12 flavours. Febs J 283: 2987-3001, 2016

95. Shackelford DB and Shaw RJ: The LKB1-AMPK pathway: Metabolism and growth control in tumour suppression. Nat Rev Cancer 9: 563-575, 2009.

96. Woods A, Dickerson K, Heath R, Hong SP, Momcilovic M, Johnstone SR, Carlson $\mathrm{M}$ and Carling D: $\mathrm{Ca}^{2+} /$ calmodulin-dependent protein kinase kinase-beta acts upstream of AMP-activated protein kinase in mammalian cells. Cell Metab 2: 21-33, 2005.

97. Ciccarese F, Zulato E and Indraccolo S: LKB1/AMPK pathway and drug response in cancer: A therapeutic perspective. Oxid Med Cell Longev 2019: 8730816, 2019.

98. Kawakami Y, Nishimoto H, Kitaura J, Maeda-Yamamoto M, Kato RM, Littman DR, Leitges M, Rawlings DJ and Kawakami T: Protein kinase $\mathrm{C}$ betaII regulates Akt phosphorylation on Ser-473 in a cell type- and stimulus-specific fashion. J Biol Chem 279: 47720-4725, 2004.

99. Partovian C and Simons M: Regulation of protein kinase B/Akt activity and Ser473 phosphorylation by protein kinase Calpha in endothelial cells. Cell Signal 16: 951-957, 2004.

100. Delbridge AR and Strasser A: The BCL-2 protein family, BH3-mimetics and cancer therapy. Cell Death Differ 22: $1071-1080,2015$.
101. Muthalagu N, Junttila MR, Wiese KE, Wolf E, Morton J, Bauer B, Evan GI, Eilers M and Murphy DJ: BIM is the primary mediator of MYC-induced apoptosis in multiple solid tissues. Cell Rep 8: 1347-1353, 2014.

102. Zhang X, Tang N, Hadden TJ and Rishi AK: Akt, FoxO and regulation of apoptosis. Biochim Biophys Acta 1813: 1978-1986, 2011

103. Cermelli S, Jang IS, Bernard B and Grandori C: Synthetic lethal screens as a means to understand and treat MYC-driven cancers. Cold Spring Harb Perspect Med 4: a014209, 2014.

104. Carling D: AMPK signalling in health and disease. Curr Opin Cell Biol 45: 31-37, 2017.

105. Jones RG, Plas DR, Kubek S, Buzzai M, Mu J, Xu Y, Birnbaum MJ and Thompson CB: AMP-activated protein kinase induces a p53-dependent metabolic checkpoint. Mol Cell 18: 283-293, 2005

106. Hou X, Liu JE, Liu W, Liu CY, Liu ZY and Sun ZY: A new role of NUAK1: Directly phosphorylating p53 and regulating cell proliferation. Oncogene 30: 2933-2942, 2011

107. Baker DJ, Jeganathan KB, Cameron JD, Thompson M, Juneja S, Kopecka A, Kumar R, Jenkins RB, de Groen PC, Roche P and van Deursen JM: BubR1 insufficiency causes early onset of aging-associated phenotypes and infertility in mice. Nat Genet 36: 744-749, 2004.

108. Chesnokova V,Zonis S,Kovacs K,Ben-Shlomo A, Wawrowsky K, Bannykh S and Melmed S: p21(Cip1) restrains pituitary tumor growth. Proc Natl Acad Sci USA 105: 17498-17503, 2004.

109. Takahashi A, Ohtani N, Yamakoshi K, Iida S, Tahara H, Nakayama K, Nakayama KI, Ide T, Saya H and Hara E: Mitogenic signalling and the p16INK4a-Rb pathway cooperate to enforce irreversible cellular senescence. Nat Cell Biol 8: 1291-1297, 2006.

110. Zhang D, Shimizu T, Araki N, Hirota T, Yoshie M, Ogawa K, Nakagata N, Takeya M and Saya H: Aurora A overexpression induces cellular senescence in mammary gland hyperplastic tumors developed in p53-deficient mice. Oncogene 27:4305-4314, 2008 .

111. Holland B, Wong J, Li M and Rasheed S: Identification of human microRNA-like sequences embedded within the protein-encoding genes of the human immunodeficiency virus. PLoS One 8: e58586, 2013

112. Obayashi M, Yoshida M, Tsunematsu T, Ogawa I, Sasahira T, Kuniyasu H, Imoto I, Abiko Y, Xu D, Fukunaga S, et al: microRNA-203 suppresses invasion and epithelial-mesenchymal transition induction via targeting NUAK1 in head and neck cancer. Oncotarget 7: 8223-8239, 2016.

113. Shenouda SK and Alahari SK: MicroRNA function in cancer: Oncogene or a tumor suppressor? Cancer Metastasis Rev 28 369-378, 2009

114. Yu Y, Wang Y, Xiao X, Chang W, Hu L, Yao W, Qian Z and Wu W: MiR-204 inhibits hepatocellular cancer drug resistance and metastasis through targeting NUAK1. Biochem Cell Biol 97: 563-570, 2019.

115. Xiong X, Sun D, Chai H, Shan W, Yu Y, Pu L and Cheng F. MiR-145 functions as a tumor suppressor targeting NUAK1 in human intrahepatic cholangiocarcinoma. Biochem Biophys Res Commun 465: 262-269, 2015.

116. Bell RE, Khaled M, Netanely D, Schubert S, Golan T, Buxbaum A, Janas MM, Postolsky B, Goldberg MS, Shamir R and Levy C: Transcription factor/microRNA axis blocks melanoma invasion program by miR-211 targeting NUAK1. J Invest Dermatol 134: 441-451, 2014.

117. Huang X, Lv W, Zhang JH and Lu DL: miR96 functions as a tumor suppressor gene by targeting NUAK1 in pancreatic cancer. Int J Mol Med 34: 1599-1605, 2014

118. Monteverde T, Tait-Mulder J, Hedley A, Knight JR, Sansom OJ and Murphy DJ: Calcium signalling links MYC to NUAK1. Oncogene 37: 982-992, 2018.

119. Ojo OO, Bhadauria S and Rath SK: Dose-dependent adverse effects of salinomycin on male reproductive organs and fertility in mice. PLoS One 8: e69086, 2013

120. Zhou Y, Liang C, Xue F, Chen W, Zhi X, Feng X, Bai X and Liang T: Salinomycin decreases doxorubicin resistance in hepatocellular carcinoma cells by inhibiting the $\beta$-catenin/TCF complex association via FOXO3a activation. Oncotarget 6 : 10350-10365, 2015

121. Yu Z, Cheng H, Zhu H, Cao M, Lu C, Bao S, Pan Y and Li Y: Salinomycin enhances doxorubicin sensitivity through reversing the epithelial-mesenchymal transition of cholangiocarcinoma cells by regulating ARK5. Braz J Med Biol Res 50: e6147, 2017 
122. Reddy MV, Akula B, Cosenza SC, Athuluridivakar S, Mallireddigari MR, Pallela VR, Billa VK, Subbaiah DR, Bharathi EV, Vasquez-Del Carpio R, et al: Discovery of 8-cyclopentyl-2-[4-(4-methyl-piperazin-1-yl)-phenylamino]-7-oxo7,8-dihydro-pyrido[2,3-d]pyrimidine-6-carbonitrile (7x) as a potent inhibitor of cyclin-dependent kinase 4 (CDK4) and AMPK-related kinase 5 (ARK5). J Med Chem 57: 578-599, 2014.

123. Huang X, Di Liberto M, Jayabalan D, Liang J, Ely S, Bretz J, Shaffer AL III, Louie T, Chen I, Randolph S, et al: Prolonged early G(1) arrest by selective CDK4/CDK6 inhibition sensitizes myeloma cells to cytotoxic killing through cell cycle-coupled loss of IRF4. Blood 120: 1095-1106, 2012.

124. Niesvizky R, Badros AZ, Costa LJ, Ely SA, Singhal SB, Stadtmauer EA, Haideri NA, Yacoub A, Hess G, Lentzsch S, et al: Phase 1/2 study of cyclin-dependent kinase (CDK) $4 / 6$ inhibitor palbociclib (PD-0332991) with bortezomib and dexamethasone in relapsed/refractory multiple myeloma. Leuk Lymphoma 56 3320-3328, 2015.

125. Banerjee S, Buhrlage SJ, Huang HT, Deng X, Zhou W, Wang J, Traynor R, Prescott AR, Alessi DR and Gray NS Characterization of WZ4003 and HTH-01-015 as selective inhibitors of the LKB1-tumour-suppressor-activated NUAK kinases. Biochem J 457: 215-225, 2014.

126. Banerjee S, Zagorska A, Deak M, Campbell DG, Prescott AR and Alessi DR: Interplay between Polo kinase, LKB1-activated NUAK1 kinase, PP1 $\beta$ MYPT1 phosphatase complex and the SCFßTrCP E3 ubiquitin ligase. Biochem J 461: 233-245, 2014.

127. Reeves WB and Andreoli TE: Transforming growth factor beta contributes to progressive diabetic nephropathy. Proc Natl Acad Sci USA 97: 7667-7669, 2000.

128. Zhang X, Lv H, Zhou Q, Elkholi R, Chipuk JE, Reddy MV, Reddy EP and Gallo JM: Preclinical pharmacological evaluation of a novel multiple kinase inhibitor, ON123300, in brain tumor models. Mol Cancer Ther 13: 1105-1116, 2014

129. Kalluri R and Weinberg RA: The basics of epithelial-mesenchymal transition. J Clin Invest 119: 1420-1428, 2009.

130. Brennan EP, Morine MJ, Walsh DW, Roxburgh SA Lindenmeyer MT, Brazil DP, Gaora PÓ, Roche HM, Sadlier DM, Cohen CD, et al: Next-generation sequencing identifies TGF- $\beta 1$-associated gene expression profiles in renal epithelial cells reiterated in human diabetic nephropathy. Biochim Biophys Acta 1822: 589-599, 2012.

131. Russo LM, del Re E, Brown D and Lin HY: Evidence for a role of transforming growth factor (TGF)-betal in the induction of postglomerular albuminuria in diabetic nephropathy: Amelioration by soluble TGF-beta type II receptor. Diabetes 56 : 380-388, 2007.
132. Sakamoto K, Goransson O, Hardie DG and Alessi DR: Activity of LKB1 and AMPK-related kinases in skeletal muscle: Effects of contraction, phenformin, and AICAR. Am J Physiol Endocrinol Metab 287: E310-E317, 2004.

133. Fisher JS, Ju JS, Oppelt PJ, Smith JL, Suzuki A and Esumi H Muscle contractions, AICAR, and insulin cause phosphorylation of an AMPK-related kinase. Am J Physiol Endocrino Metab 289: E986-E992, 2005.

134. Hoppe PE, Chau J, Flanagan KA, Reedy AR and Schriefer LA: Caenorhabditis elegans unc-82 encodes a serine/threonine kinase important for myosin filament organization in muscle during growth. Genetics 184: 79-90, 2010.

135. Zagorska A, Deak M, Campbell DG, Banerjee S, Hirano M, Aizawa S, Prescott AR and Alessi DR: New roles for the LKB1-NUAK pathway in controlling myosin phosphatase complexes and cell adhesion. Sci Signal 3: ra25, 2010.

136. Inazuka F, Sugiyama N, Tomita M, Abe T, Shioi G and Esumi H: Muscle-specific knock-out of NUAK family SNF1-like kinase 1 (NUAK1) prevents high fat diet-induced glucose intolerance. J Biol Chem 287: 16379-16389, 2012

137. Yap JKY, Pickard BS, Chan EWL and Gan SY: The role of neuronal NLRP1 inflammasome in Alzheimer's disease: Bringing neurons into the neuroinflammation game. Mol Neurobiol 56: 7741-7753, 2019.

138. Courchet V, Roberts AJ, Meyer-Dilhet G, Del Carmine P, Lewis TL Jr, Polleux F and Courchet J: Haploinsufficiency of autism spectrum disorder candidate gene NUAK1 impairs cortical development and behavior in mice. Nat Commun 9: 4289, 2018.

139. Soto C and Pritzkow S: Protein misfolding, aggregation, and conformational strains in neurodegenerative diseases. Nat Neurosci 21: 1332-1340, 2018.

140. Cao Q, Wang XJ, Liu CW, Liu DF, Li LF, Gao YQ and Su XD Inhibitory mechanism of caspase- 6 phosphorylation revealed by crystal structures, molecular dynamics simulations, and biochemical assays. J Biol Chem 287: 15371-15379, 2012.

141. MacLachlan TK and El-Deiry WS: Apoptotic threshold is lowered by 553 transactivation of caspase-6. Proc Natl Acad Sci USA 99: 9492-9497, 2002

142. Kurokawa M and Kornbluth S: Caspases and kinases in a death grip. Cell 138: 838-854, 2009

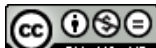

This work is licensed under a Creative Commons Attribution-NonCommercial-NoDerivatives 4.0 International (CC BY-NC-ND 4.0) License. 